\title{
On canonical constructions on connections
}

\begin{abstract}
We study how a projectable general connection $\Gamma$ in a 2-fibred manifold $Y^{2} \rightarrow Y^{1} \rightarrow Y^{0}$ and a general vertical connection $\Theta$ in $Y^{2} \rightarrow Y^{1} \rightarrow$ $Y^{0}$ induce a general connection $A(\Gamma, \Theta)$ in $Y^{2} \rightarrow Y^{1}$.
\end{abstract}

Introduction. In Section 1, we introduce the concepts of projectable general connections $\Gamma$ and general vertical connections $\Theta$ in a 2-fibred manifold $Y^{2} \rightarrow Y^{1} \rightarrow Y^{0}$. In Section 2, we construct a general connection $\Sigma(\Gamma, \Theta)$ in $Y^{2} \rightarrow Y^{1}$ from a projectable general connection $\Gamma$ in $Y^{2} \rightarrow Y^{1} \rightarrow Y^{0}$ by means of a general vertical connection $\Theta$ in $Y^{2} \rightarrow Y^{1} \rightarrow Y^{0}$. In Section 3 we observe the canonical character of the construction $\Sigma(\Gamma, \Theta)$. In Section 4 , we cite the concepts of natural operators. In Section 5, we describe completely the natural operators $A$ transforming tuples $(\Gamma, \Theta)$ as above into general connections $A(\Gamma, \Theta)$ in $Y^{2} \rightarrow Y^{1}$. In Section 6 , we prove that there is no natural operator $C$ producing general connections $C(\Gamma)$ in $Y^{2} \rightarrow Y^{1}$ from projectable general connections $\Gamma$ in $Y^{2} \rightarrow Y^{1} \rightarrow Y^{0}$. In Section 7 , we present a construction of a general connection $\Sigma(\Gamma, \Theta)$ in $Y^{2} \rightarrow Y^{1}$ from a system $\Gamma=\left(\Gamma^{2}, \Gamma^{1}\right)$ of a general connection $\Gamma^{2}$ in $Y^{2} \rightarrow Y^{0}$ and a general connection $\Gamma^{1}$ in $Y^{1} \rightarrow Y^{0}$ by means of a general vertical connection $\Theta$ in $Y^{2} \rightarrow Y^{1} \rightarrow Y^{0}$. In Section 8, we present an application of the obtained result in prolongation of general connections to bundle functors.

2010 Mathematics Subject Classification. 53C05, 58A32.

Key words and phrases. General connection, projectable general connection, general vertical connection, 2-fibred manifold, natural operator. 
All manifolds considered in the note is Hausdorff, second countable, without boundaries, finite dimensional and smooth (of class $C^{\infty}$ ). Maps between manifolds are smooth (infinitely differentiable).

1. Connections. A fibred manifold is a surjective submersion $p: Y \rightarrow M$ between manifolds. By [1], an $r$-th order holonomic connection in $p: Y \rightarrow$ $M$ is a section

$$
\Gamma: Y \rightarrow J^{r} Y
$$

of the holonomic $r$-jet prolongation $\pi_{0}^{r}: J^{r} Y \rightarrow Y$ of $Y \rightarrow M$. If $Y \rightarrow M$ is a vector bundle and $\Gamma: Y \rightarrow J^{r} Y$ is a vector bundle map, $\Gamma$ is called a linear $r$-th order holonomic connection in $Y \rightarrow M$. A linear $r$-th order holonomic connection in the tangent bundle $Y=T M \rightarrow M$ of $M$ is called an $r$-th order linear connection on $M$. A first order linear connection on $M$ is in fact a classical linear connection on $M$.

A 1-order holonomic connection $\Gamma: Y \rightarrow J^{1} Y$ in a fibred manifold $Y \rightarrow$ $M$ is called a general connection in $Y \rightarrow M$.

We have the following equivalent definitions of general connections in $Y \rightarrow M$, see [1].

A general connection in $p: Y \rightarrow M$ is a lifting map

$$
\Gamma: Y \times_{M} T M \rightarrow T Y,
$$

i.e. a vector bundle map covering the identity map $i d_{Y}: Y \rightarrow Y$ such that

$$
T p \circ \Gamma(y, w)=w
$$

for any $y \in Y_{x}, w \in T_{x} M, x \in M$. (More precisely, $\Gamma(y, w)=T_{x} \sigma(w)$, where $\Gamma(y)=j_{x}^{1} \sigma$.)

A general connection in $Y \rightarrow M$ is a vector bundle decomposition

$$
T Y=V Y \oplus_{Y} H^{\Gamma}
$$

of the tangent bundle $T Y$ of $Y$, where $V Y$ is the vertical bundle of $Y$. (More precisely, $H_{y}^{\Gamma}=\operatorname{im} T_{x} \sigma$, where $\Gamma(y)=j_{x}^{1} \sigma$.) $\left.H^{\Gamma}\right)$

A general connection in $Y \rightarrow M$ is a vector bundle projection (in direction covering $i d_{Y}$.

$$
p r^{\Gamma}: T Y \rightarrow V Y
$$

A 2-fibred manifold is a system $Y^{2} \rightarrow Y^{1} \rightarrow Y^{0}$ of two fibred manifolds $Y^{2} \rightarrow Y^{1}$ and $Y^{1} \rightarrow Y^{0}$.

Let $Y^{2} \rightarrow Y^{1} \rightarrow Y^{0}$ be 2-fibred manifold and

$$
p^{i j}: Y^{i} \rightarrow Y^{j}, 0 \leq j<i \leq 2
$$

be its projections. Of course, $p^{20}=p^{10} \circ p^{21}$. Let

$$
V^{i j} Y^{i}:=\operatorname{ker}\left(T p^{i j}: T Y^{i} \rightarrow T Y^{j}\right)
$$

be the vertical bundle of $p^{i j}: Y^{i} \rightarrow Y^{j}, 0 \leq j<i \leq 2$. 
We introduce the following concepts of projectable general connections and of general vertical connections in 2-fibred manifolds $Y^{2} \rightarrow Y^{1} \rightarrow Y^{0}$.

A projectable general connection in $Y^{2} \rightarrow Y^{1} \rightarrow Y^{0}$ is a general connection

$$
\Gamma: Y^{2} \times_{Y^{0}} T Y^{0} \rightarrow T Y^{2}
$$

in $p^{20}: Y^{2} \rightarrow Y^{0}$ such that there is a (unique) general connection

$$
\underline{\Gamma}: Y^{1} \times_{Y^{0}} T Y^{0} \rightarrow T Y^{1}
$$

in $p^{10}: Y^{1} \rightarrow Y^{0}$ satisfying

$$
T p^{21} \circ \Gamma=\underline{\Gamma} \circ\left(p^{21} \times i d_{T Y^{0}}\right) .
$$

Connection $\underline{\Gamma}$ is called the underlying connection of $\Gamma$.

A general vertical connection in $Y^{2} \rightarrow Y^{1} \rightarrow Y^{0}$ is a vector bundle map

$$
\Theta: Y^{2} \times_{Y^{1}} V^{10} Y^{1} \rightarrow V^{20} Y^{2}
$$

covering the identity map $i d_{Y^{2}}: Y^{2} \rightarrow Y^{2}$ such that

$$
T p^{21} \circ \Theta\left(y^{2}, v^{1}\right)=v^{1}
$$

for any $y^{2} \in Y_{y^{1}}^{2}, y^{1} \in Y^{1}$ and $v^{1} \in V_{y^{1}}^{10} Y^{1}$.

Equivalently, a general vertical connection in $Y^{2} \rightarrow Y^{1} \rightarrow Y^{0}$ is a smoothly parametrized system $\Theta=\left(\Theta_{x}\right)$ of general connections

$$
\Theta_{x}: Y_{x}^{2} \times_{Y_{x}^{1}} T Y_{x}^{1} \rightarrow T Y_{x}^{2}
$$

in the fibred manifolds $Y_{x}^{2} \rightarrow Y_{x}^{1}$ for any $x \in Y^{0}$, where $Y_{x}^{2}$ is the fibre of $p^{20}: Y^{2} \rightarrow Y^{0}$ over $x$ and $Y_{x}^{1}$ is the fibre of $p^{10}: Y^{1} \rightarrow Y^{0}$ over $x$ and $Y_{x}^{2} \rightarrow Y_{x}^{1}$ is the restriction of the projection $p^{21}: Y^{2} \rightarrow Y^{1}$.

2. A construction. Let $\Gamma$ be a projectable general connection in $Y^{2} \rightarrow$ $Y^{1} \rightarrow Y^{0}$ with the underlying connection $\underline{\Gamma}$ and $\Theta$ be a general vertical connection in $Y^{2} \rightarrow Y^{1} \rightarrow Y^{0}$.

We define a map $\Sigma(\Gamma, \Theta)=\Sigma: Y^{2} \times_{Y^{1}} T Y^{1} \rightarrow T Y^{2}$ by

$$
\Sigma\left(y^{2}, w^{1}\right):=\Theta\left(y^{2}, p r^{\Gamma}\left(w^{1}\right)\right)+\Gamma\left(y^{2}, T p^{10}\left(w^{1}\right)\right),
$$

$y^{2} \in Y_{y^{1}}^{2}, y^{1} \in Y^{1}, w^{1} \in T_{y^{1}} Y^{1}$, where $p r \underline{\Gamma}: T Y^{1} \rightarrow V^{10} Y^{1}$ is the -projection.

Lemma 1. $\Sigma$ is a general connection in $p^{21}: Y^{2} \rightarrow Y^{1}$.

Proof. It is sufficient to verify that $T p^{21} \circ \Sigma\left(y^{2}, w^{1}\right)=w^{1}$. We consider two cases.

(a) Let $w^{1} \in V_{y^{1}}^{10} Y^{1}$. Then $\Sigma\left(y^{2}, w^{1}\right)=\Theta\left(y^{2}, w^{1}\right)$, and then

$$
T p^{21} \circ \Sigma\left(y^{2}, w^{1}\right)=T p^{21} \circ \Theta\left(y^{2}, w^{1}\right)=w^{1}
$$

as $\Theta$ is a general vertical connection in $Y^{2} \rightarrow Y^{1} \rightarrow Y^{0}$. 
(b) Let $w^{1} \in H_{y^{1}}^{\Gamma} Y^{1}$, the $\underline{\Gamma-h o r i z o n t a l}$ space. Denote $w^{0}=T p^{10}\left(w^{1}\right)$. Then $\Sigma\left(y^{2}, w^{1}\right)=\Gamma\left(y^{2}, w^{0}\right)$, and then

$$
T p^{21} \circ \Sigma\left(y^{2}, w^{1}\right)=T p^{21} \circ \Gamma\left(y^{2}, w^{0}\right)=\underline{\Gamma}\left(p^{21}\left(y^{2}\right), w^{0}\right)=\underline{\Gamma}\left(y^{1}, w^{0}\right) .
$$

Then $w^{\prime}:=T p^{21} \circ \Sigma\left(y^{2}, w^{1}\right) \in H_{y^{1}}^{\Gamma} Y^{1}, w^{1} \in H_{y^{1}}^{\Gamma} Y^{1}$ and

$$
T p^{10}\left(w^{\prime}\right)=T p^{10} \circ T p^{21} \circ \Gamma\left(y^{2}, w^{0}\right)=T p^{20} \circ \Gamma\left(y^{2}, w^{0}\right)=w^{0}=T p^{10}\left(w^{1}\right),
$$

and consequently $w^{\prime}=w^{1}$.

3. Invariance. Let $\tilde{Y}^{2} \rightarrow \tilde{Y}^{1} \rightarrow \tilde{Y}^{0}$ be another 2-fibred manifold with projections $\tilde{p}^{i j}: \tilde{V}^{i} \rightarrow \tilde{V}^{j}, 0 \leq j<i \leq 2$. Let $\tilde{\Gamma}$ be a projectable general connection in $\tilde{Y}^{2} \rightarrow \tilde{Y}^{1} \rightarrow \tilde{Y}^{0}$ and $\tilde{\Theta}$ be a general vertical connection in $\tilde{Y}^{2} \rightarrow \tilde{Y}^{1} \rightarrow \tilde{Y}^{0}$. Let $f=\left(f^{2}, f^{1}, f^{0}\right):\left(Y^{2} \rightarrow Y^{1} \rightarrow Y^{0}\right) \rightarrow\left(\tilde{Y}^{2} \rightarrow \tilde{Y}^{1} \rightarrow\right.$ $\left.\tilde{Y}^{0}\right)$ be a 2-fibred map, i.e. $f^{i}: Y^{i} \rightarrow \tilde{Y}^{i}$ for $i=0,1,2$ and $\tilde{p}^{i j} \circ f^{i}=f^{j} \circ p^{i j}$ for $0 \leq j<i \leq 2$.

Lemma 2. If $\Gamma$ is f-related with $\tilde{\Gamma}$, (i.e. $T f^{2} \circ \Gamma=\tilde{\Gamma} \circ\left(f^{2} \times_{f^{0}} T f^{0}\right)$ and then $\left.T f^{1} \circ \underline{\Gamma}=\tilde{\Gamma} \circ\left(f^{1} \times f_{0} T f^{0}\right)\right)$ and $\Theta$ is f-related with $\tilde{\Theta}$ (i.e. $V^{20} f^{2} \circ \Theta=$ $\left.\tilde{\Theta} \circ\left(f^{2} \times f^{1} V^{10} f^{1}\right)\right)$, then $\Sigma=\Sigma(\Gamma, \Theta)$ is f-related with $\tilde{\Sigma}=\Sigma(\tilde{\Gamma}, \tilde{\Theta})$ (i.e. $\left.T f^{2} \circ \Sigma=\tilde{\Sigma} \circ\left(f^{2} \times f^{1} T f^{1}\right)\right)$.

Proof. If $w \in H^{\Gamma} Y^{1}$, then $w=\underline{\Gamma}\left(y^{1}, w^{0}\right)$ for some $y^{1} \in Y_{y^{0}}^{1}$ and $w^{0} \in Y_{y^{0}}^{0}$, and then $T f^{1}(w)=\underline{\tilde{\Gamma}}\left(f^{1}\left(y^{1}\right), T f^{0}\left(w^{0}\right)\right) \in H^{\tilde{\Gamma}}$. Then

$$
T f^{1}\left(H^{\underline{\Gamma}} Y^{1}\right) \subset H^{\tilde{\Gamma}} \tilde{Y}^{1} \text { and (obviously) } T f^{1}\left(V^{10} Y^{1}\right) \subset V^{10} \tilde{Y}^{1} .
$$

Consequently, $V^{10} f^{1} \circ p r \underline{\underline{\Gamma}}=p r \underline{\tilde{\Gamma}} \circ T f^{1}$. Using this formula and the assumption of the lemma and the formula defining $\Sigma$, one can easily verify that

$$
T f^{2} \circ \Sigma\left(y^{2}, w^{1}\right)=\tilde{\Sigma} \circ\left(f^{2}\left(y^{2}\right), T f^{1}\left(w^{1}\right)\right)
$$

for $y^{2} \in Y_{y^{1}}^{2}, w^{1} \in T_{y^{1}} Y^{1}, y^{1} \in Y^{1}$.

4. Natural operators. The general concept of natural operators can be found in [1]. We need the following partial cases of this general concept.

Let $\mathcal{F} \mathcal{M}_{m_{0}, m_{1}, m_{2}}$ be the category of 2-fibred manifolds $Y^{2} \rightarrow Y^{1} \rightarrow Y^{0}$ with $\operatorname{dim}\left(Y^{0}\right)=m_{0}, \operatorname{dim}\left(Y^{1}\right)=m_{0}+m_{1}, \operatorname{dim}\left(Y^{2}\right)=m_{0}+m_{1}+m_{2}$ and their 2-fibred local diffeomorphisms.

Definition 1. An $\mathcal{F} \mathcal{M}_{m_{0}, m_{1}, m_{2}}$-natural operator transforming projectable general connections $\Gamma$ and general vertical connections $\Theta$ in $\mathcal{F} \mathcal{M}_{m_{0}, m_{1}, m_{2}}{ }^{-}$ objects $Y^{2} \rightarrow Y^{1} \rightarrow Y^{0}$ into general connections $A(\Gamma, \Theta)$ in $Y^{2} \rightarrow Y^{1}$ is an $\mathcal{F} \mathcal{M}_{m_{0}, m_{1}, m_{2}}$-invariant system $A$ of regular operators (functions)

$A: \operatorname{Con}_{\text {proj }}\left(Y^{2} \rightarrow Y^{1} \rightarrow Y^{0}\right) \times \operatorname{Con}_{\text {vert }}\left(Y^{2} \rightarrow Y^{1} \rightarrow Y^{0}\right) \rightarrow \operatorname{Con}\left(Y^{2} \rightarrow Y^{1}\right)$ 


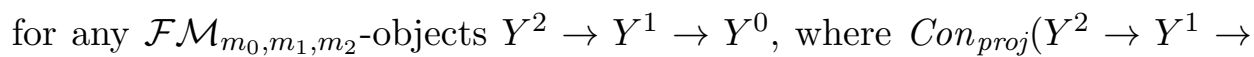
$\left.Y^{0}\right)$ is the set of projectable general connections in $Y^{2} \rightarrow Y^{1} \rightarrow Y^{0}$, Con $_{\text {vert }}\left(Y^{2} \rightarrow Y^{1} \rightarrow Y^{0}\right)$ is the set of general vertical connections in $Y^{2} \rightarrow Y^{1} \rightarrow Y^{0}$ and $\operatorname{Con}\left(Y^{2} \rightarrow Y^{1}\right)$ is the set of general connections in $Y^{2} \rightarrow Y^{1}$.

The invariance of $A$ means that if $\Gamma \in \operatorname{Con}_{\text {proj }}\left(Y^{2} \rightarrow Y^{1} \rightarrow Y^{0}\right)$ is $f$ related with $\tilde{\Gamma} \in \operatorname{Con}_{\text {proj }}\left(\tilde{Y}^{2} \rightarrow \tilde{Y}^{1} \rightarrow \tilde{Y}^{0}\right)$ and $\Theta \in \tilde{C o n}_{\text {vert }}\left(Y^{2} \rightarrow Y^{1} \rightarrow\right.$ $\left.Y^{0}\right)$ is $f$-related with $\tilde{\Theta} \in \operatorname{Con}_{\text {vert }}\left(\tilde{Y}^{2} \rightarrow \tilde{Y}^{1} \rightarrow \tilde{Y}^{0}\right)$ for an $\mathcal{F} \mathcal{M}_{m_{0}, m_{1}, m_{2}}{ }^{-}$ morphism $f=\left(f^{2}, f^{1}, f^{0}\right):\left(Y^{2} \rightarrow Y^{1} \rightarrow Y^{0}\right) \rightarrow\left(\tilde{Y}^{2} \rightarrow \tilde{Y}^{1} \rightarrow \tilde{Y}^{0}\right)$, then $A(\Gamma, \Theta)$ is $f$-related with $A(\tilde{\Gamma}, \tilde{\Theta})$.

The regularity of $A$ means that $A$ transforms smoothly parametrized families into smoothly parametrized families.

Because of Lemma 2, the construction $\Sigma(\Gamma, \Theta)$ defines an $\mathcal{F} \mathcal{M}_{m_{0}, m_{1}, m_{2}}{ }^{-}$ natural operator in the sense of Definition 1. So, to describe all natural operators $A$ in the sense of Definition 1 it is sufficient to describe all natural operators in the sense of the following definition.

Definition 2. An $\mathcal{F} \mathcal{M}_{m_{0}, m_{1}, m_{2}}$-natural operator transforming projectable general connections $\Gamma$ and general vertical connections $\Theta$ in $\mathcal{F} \mathcal{M}_{m_{0}, m_{1}, m_{2}}{ }^{-}$ objects $Y^{2} \rightarrow Y^{1} \rightarrow Y^{0}$ into sections $B(\Gamma, \Theta): Y^{2} \rightarrow T^{*} Y^{1} \otimes V^{21} Y^{2}$ of $T^{*} Y^{1} \otimes V^{21} Y^{2} \rightarrow Y^{2}$ is an $\mathcal{F} \mathcal{M}_{m_{0}, m_{1}, m_{2}}$-invariant system $A$ of regular operators

$B: \operatorname{Con}_{\text {proj }}\left(Y^{2} \rightarrow Y^{1} \rightarrow Y^{0}\right) \times \operatorname{Con}_{\text {vert }}\left(Y^{2} \rightarrow Y^{1} \rightarrow Y^{0}\right) \rightarrow C_{Y^{2}}^{\infty}\left(T^{*} Y^{1} \otimes V^{21} Y^{2}\right)$

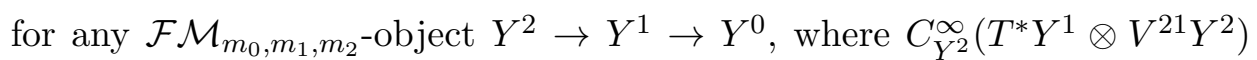
is the space of sections of the vector bundle $T^{*} Y^{1} \otimes V^{21} Y^{2}$ over $Y^{2}$ (with respect to the clear projection).

It is obvious that any natural operator $A$ in the sense of Definition 1 is of the form

$$
A(\Gamma, \Theta)=\Sigma(\Gamma, \Theta)+B(\Gamma, \Theta)
$$

for a uniquely determined (by $A$ ) natural operator $B$ in the sense of Definition 2.

A simple example of a natural operator in the sense of Definition 2 is the one $B^{o}$ defined by

$$
B^{o}(\Gamma, \Theta)\left(y^{2}\right)\left(w^{1}\right)=p r^{\Sigma(\Gamma, \Theta)} \circ \Theta\left(y^{2}, p r^{\Gamma}\left(w^{1}\right)\right) \in V_{y^{2}}^{21} Y^{2}
$$

for any $\mathcal{F} \mathcal{M}_{m_{0}, m_{1}, m_{2}}$-object $Y^{2} \rightarrow Y^{1} \rightarrow Y^{0}, \Gamma \in \operatorname{Con}_{\text {proj }}\left(Y^{2} \rightarrow Y^{1} \rightarrow Y^{0}\right)$, $\Theta \in \operatorname{Con}_{\text {vert }}\left(Y^{2} \rightarrow Y^{1} \rightarrow Y^{0}\right), y^{2} \in Y_{y^{1}}^{2}, y^{1} \in Y^{1}, w^{1} \in T_{y^{1}} Y^{1}$, where $p^{\Sigma(\Gamma, \Theta)}: T Y^{2} \rightarrow V^{21} Y^{2}$ is the $\Sigma(\Gamma, \Theta)$-projection. 
5. A classification. Let $\mathbf{R}^{m_{0}, m_{1}, m_{2}}$ be the trivial $\mathcal{F} \mathcal{M}_{m_{0}, m_{1}, m_{2} \text {-object }}$ $\mathbf{R}^{m_{0}} \times \mathbf{R}^{m_{1}} \times \mathbf{R}^{m_{2}} \rightarrow \mathbf{R}^{m_{0}} \times \mathbf{R}^{m_{1}} \rightarrow \mathbf{R}^{m_{0}}$ with the usual projections. Let $x^{1}, \ldots, x^{m_{0}}, y^{1}, \ldots, y^{m_{1}}, z^{1}, \ldots, z^{m_{2}}$ be the usual coordinates on $\mathbf{R}^{m_{0}, m_{1}, m_{2}}$.

Consider a natural operator $B$ in the sense of Definition 2. Because of the invariance of $B$ with respect to 2 -fibred manifold charts, $B$ is determined by the linear maps

$$
B(\Gamma, \Theta)(0,0,0): T_{(0,0)}\left(\mathbf{R}^{m_{0}} \times \mathbf{R}^{m_{1}}\right) \rightarrow V_{(0,0,0)}^{21}\left(\mathbf{R}^{m_{0}} \times \mathbf{R}^{m_{1}} \times \mathbf{R}^{m_{2}}\right)
$$

for all $\Gamma \in \operatorname{Con}_{\text {proj }}\left(\mathbf{R}^{m_{0}, m_{1}, m_{2}}\right)$ and all $\Theta \in \operatorname{Con}_{\text {vert }}\left(\mathbf{R}^{m_{0}, m_{1}, m_{2}}\right)$ of the forms

$$
\begin{gathered}
\Gamma=\Gamma^{o}+\sum \Gamma_{i}^{p}(x, y) d x^{i} \otimes \frac{\partial}{\partial y^{p}}+\sum \Gamma_{i}^{q}(x, y, z) d x^{i} \otimes \frac{\partial}{\partial z^{q}}, \\
\Theta=\Theta^{o}+\sum \Theta_{p}^{q}(x, y, z) d y^{p} \otimes \frac{\partial}{\partial z^{q}},
\end{gathered}
$$

where the sums are over $i=1, \ldots, m_{0}, p=1, \ldots, m_{1}, q=1, \ldots, m_{2}$, and where $\Gamma^{o}$ denotes the trivial projectable general connection in $\mathbf{R}^{m_{0}, m_{1}, m_{2}}$ and $\Theta^{o}=\sum d y^{p} \otimes \frac{\partial}{\partial y^{p}}$ denotes the trivial general vertical connection in $\mathbf{R}^{m_{0}, m_{1}, m_{2}}$

Eventually, using a new 2-fibred manifold chart one can additionally assume $\Gamma_{i}^{p}(0,0)=0$ and $\Gamma_{i}^{q}(0,0,0)=0$. (More precisely, denote $j_{0}^{1} \sigma:=$ $\Gamma(0,0,0)$ and $\sigma(x)=:(x, \tilde{\sigma}(x), \bar{\sigma}(x))$. We consider the 2-fibred coordinate system $(x, y-\tilde{\sigma}(x), z-\bar{\sigma}(x))$. In the coordinate system $\Gamma(0,0,0)=$ $\Gamma^{o}(0,0,0)$.)

Then using the invariance of $B$ with respect to $\mathcal{F} \mathcal{M}_{m_{0}, m_{1}, m_{2}}$-map $\frac{1}{t}$ id for $t>0$ and then putting $t \rightarrow 0$, we can assume $\Gamma=\Gamma^{o}$ and $\Theta_{p}^{q}(x, y, z)=$ $\Theta_{p}^{q}(0,0,0)=$ const. Consequently, $B$ is determined by the maps

$$
B\left(\Gamma^{o}, \Theta^{o}+\sum \Theta_{p}^{q} d y^{p} \otimes \frac{\partial}{\partial z^{q}}\right)(0,0,0): \mathbf{R}^{m_{0}} \times \mathbf{R}^{m_{1}} \rightarrow \mathbf{R}^{m_{2}}
$$

for all $\Theta_{p}^{q} \in \mathbf{R}, p=1, \ldots, m_{1}, q=1, \ldots, m_{2}$.

Using the invariance of $B$ with respect to $t i d_{\mathbf{R}^{m_{0}}} \times i d_{\mathbf{R}^{m_{1}}} \times i d_{\mathbf{R}^{m_{2}}}$ and then putting $t \rightarrow 0$, we deduce that $B\left(\Gamma^{o}, \Theta^{o}+\sum \Theta_{p}^{q} d y^{p} \otimes \frac{\partial}{\partial z^{q}}\right)(0,0,0)$ do not depend on elements from $\mathbf{R}^{m_{0}}$. Consequently, $B$ is determined by the map $\Phi: \mathbf{R}^{m_{1}^{*}} \otimes \mathbf{R}^{m_{2}} \rightarrow \mathbf{R}^{m_{1}^{*}} \otimes \mathbf{R}^{m_{2}}$ given by

$$
\Phi\left(\left(\Theta_{p}^{q}\right)\right)=B\left(\Gamma^{o}, \Theta^{o}+\sum \Theta_{p}^{q} d y^{p} \otimes \frac{\partial}{\partial z^{q}}\right)(0,0,0) \in \mathbf{R}^{m_{1}^{*}} \otimes \mathbf{R}^{m_{2}} .
$$

Using the invariance of $B$ with respect to linear isomorphisms from $\left\{i d_{\mathbf{R}^{m_{0}}}\right\} \times G L\left(m_{1}\right) \times G L\left(m_{2}\right)$, we deduce that $\Phi$ is $G L\left(m_{1}\right) \times G L\left(m_{2}\right)$ invariant. Consequently, $\Phi$ is the constant multiple of the identity. Then the space of all $\mathcal{F} \mathcal{M}_{m_{0}, m_{1}, m_{2}}$-natural operators $B$ in the sense of Definition 2 is 1-dimensional. So, any natural operator $B$ in the sense of Definition 2 is the constant multiple of $B^{o}$.

Thus we proved the following classification theorem. 
Theorem 1. Any $\mathcal{F M}_{m_{0}, m_{1}, m_{2}}$-natural operator $A$ in the sense of Definition 1 is of the form

$$
A(\Gamma, \Theta)=\Sigma(\Gamma, \Theta)+\tau B^{o}(\Gamma, \Theta)
$$

for a uniquely (by $A$ ) real number $\tau$.

6. Why do we use auxiliary a general vertical connection? We prove the following theorem.

Theorem 2. There is no $\mathcal{F} \mathcal{M}_{m_{0}, m_{1}, m_{2}}$-natural operator

$$
C: \operatorname{Con}_{\text {proj }}\left(Y^{2} \rightarrow Y^{1} \rightarrow Y^{0}\right) \rightarrow \operatorname{Con}\left(Y^{2} \rightarrow Y^{1}\right)
$$

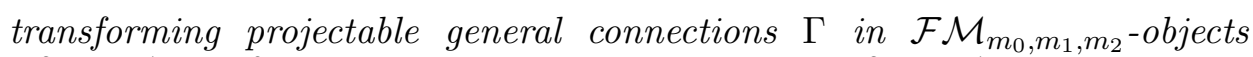
$Y^{2} \rightarrow Y^{1} \rightarrow Y^{0}$ into general connections $C(\Gamma)$ in $Y^{2} \rightarrow Y^{1}$.

Proof. Suppose that such $C$ exists. Let $\Gamma^{o}$ be the trivial projectable general connection in the 2-fibred manifold $\mathbf{R}^{m_{0}, m_{1}, m_{2}}$. Then $C\left(\Gamma^{o}\right)$ is $\varphi$-invariant by any $\mathcal{F} \mathcal{M}_{m_{0}, m_{1}, m_{2}}$-map $\varphi$ of the form $\varphi\left(x_{0}, x_{1}, x_{2}\right)=\left(x_{0}, \varphi_{1}\left(x_{1}\right), \varphi_{2}\left(x_{1}, x_{2}\right)\right)$, $x_{0} \in \mathbf{R}^{m_{0}}, x_{1} \in \mathbf{R}^{m_{1}}, x_{2} \in \mathbf{R}^{m_{2}}$ (as $\Gamma^{o}$ is). Then $j_{(0,0)}^{1} \sigma:=C\left(\Gamma^{o}\right)(0,0,0)$ is $\varphi$-invariant for any $\varphi$ as above with $\varphi(0,0,0)=(0,0,0)$. Then for $\varphi_{1}\left(x_{1}\right)=$ $x_{1}$ and $\varphi_{2}\left(x_{1}, x_{2}\right)=x_{2}+\left(x_{1}^{1}, 0, \ldots, 0\right)$ we get $j_{(0,0)}^{1}(\varphi \circ \sigma)=j_{(0,0)}^{1} \sigma$, i.e. $j_{(0,0)}^{1} \eta=0$, where $\eta\left(x_{0}, x_{1}\right)=\left(x_{0}, x_{1}, x_{1}^{1}, 0, \ldots, 0\right)$. Contradiction.

So, to construct canonically a general connection in $Y^{2} \rightarrow Y^{1}$ from a projectable general connection in $Y^{2} \rightarrow Y^{1} \rightarrow Y^{0}$ the using of auxiliary objects is unavoidable. In the present note we have used general vertical connections as such auxiliary ones.

7. A generalization. Let $Y^{2} \rightarrow Y^{1} \rightarrow Y^{0}$ be a 2-fibred manifold.

A projectable general connection $\Gamma$ in $Y^{2} \rightarrow Y^{1} \rightarrow Y^{0}$ is in fact a system $\Gamma=(\Gamma, \underline{\Gamma})$ of two general connections in $p^{20}: Y^{2} \rightarrow Y^{0}$ and $p^{10}: Y^{1} \rightarrow Y^{0}$ (respectively), and $\underline{\Gamma}$ is determined by $\Gamma$.

In this section, we present how to extend the construction of $\Sigma(\Gamma, \Theta)$ for $\Gamma=(\Gamma, \underline{\Gamma})$ into a construction $\Sigma(\Gamma, \Theta)$ for $\Gamma=\left(\Gamma^{2}, \Gamma^{1}\right)$, where $\Gamma^{2}$ : $Y^{2} \times_{Y^{0}} T Y^{0} \rightarrow T Y^{2}$ is a general connection in $p^{20}: Y^{2} \rightarrow Y^{0}$ and $\Gamma^{1}:$ $Y^{1} \times_{Y^{0}} T Y^{0} \rightarrow T Y^{1}$ is a general connection in $p^{10}: Y^{1} \rightarrow Y^{0}$.

Let $\Gamma=\left(\Gamma^{2}, \Gamma^{1}\right)$ and $\Theta$ be in question. We define a map $\Sigma(\Gamma, \Theta)=\Sigma$ : $Y^{2} \times_{Y^{1}} T Y^{1} \rightarrow T Y^{2}$ by

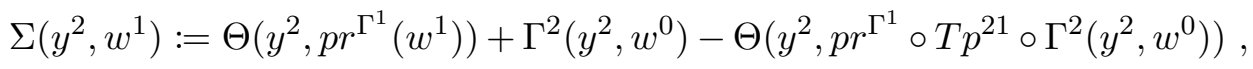
$y^{2} \in Y_{y^{1}}^{2}, y^{1} \in Y^{1}, w^{1} \in T_{y^{1}} Y^{1}, w^{0}=T p^{10}\left(w^{1}\right)$.

Lemma 3. $\Sigma$ is a general connection in $p^{21}: Y^{2} \rightarrow Y^{1}$.

Proof. We are going to prove that $T p^{21} \circ \Sigma\left(y^{2}, w^{1}\right)=w^{1}$. We consider two cases. 
(a) Let $w^{1} \in V_{y^{1}}^{10} Y^{1}$. Then $\Sigma\left(y^{2}, w^{1}\right)=\Theta\left(y^{2}, w^{1}\right)$, and next we proceed as in the part (a) of the proof of Lemma 1.

(b) Let $w^{1} \in H_{y^{1}}^{\Gamma^{1}} Y^{1}$. Then

$$
\Sigma\left(y^{2}, w^{1}\right)=\Gamma^{2}\left(y^{2}, w^{0}\right)-\Theta\left(y^{2}, p r^{\Gamma^{1}} \circ T p^{21} \circ \Gamma^{2}\left(y^{2}, w^{0}\right)\right),
$$

and then

$$
T p^{21} \circ \Sigma\left(y^{2}, w^{1}\right)=T p^{21} \circ \Gamma^{2}\left(y^{2}, w^{0}\right)-p r^{\Gamma^{1}} \circ T p^{21} \circ \Gamma^{2}\left(y^{2}, w^{0}\right) .
$$

So, $w^{\prime}:=T p^{21} \circ \Sigma\left(y^{2}, w^{1}\right) \in H_{y^{1}}^{\Gamma^{1}} Y^{1}$ and $w^{1} \in H_{y^{1}}^{\Gamma^{1}} Y^{1} \in H_{y^{1}}^{\Gamma^{1}} Y^{1}$ and

$$
T p^{10}\left(w^{\prime}\right)=T p^{20} \circ \Gamma^{2}\left(y^{2}, w^{0}\right)-0=w^{0}=T p^{10}\left(w^{1}\right),
$$

and consequently $w^{\prime}=w^{1}$.

8. An application. We can use the construction $\Sigma(\Gamma, \Theta)$ from the previous section in prolongation of connections to bundle functors.

Namely, let $F: \mathcal{F} \mathcal{M}_{m, n} \rightarrow \mathcal{F} \mathcal{M}$ be a bundle functor in the sense of [1] of order $r$, where $\mathcal{F} \mathcal{M}$ is the category of fibred manifolds and fibred maps and $\mathcal{F} \mathcal{M}_{m, n}$ is the category of fibred manifolds with $m$-dimensional bases and $n$-dimensional fibres and their local fibred diffeomorphisms. Let $p: Y \rightarrow M$ be an $\mathcal{F} \mathcal{M}_{m, n}$-object. Let $\Xi$ be a general connection in $p: Y \rightarrow M$ and $\lambda$ be an $r$-th order linear connection on $M$ (i.e. $r$-th order linear connection in $T M \rightarrow M$ ). Thus we have the $F$-prolongation $\mathcal{F}(\Xi, \lambda)$ (of $\Xi$ with respect to $\lambda)$ in the sense of $[1$, Def. 45.4]. $\mathcal{F}(\Xi, \lambda)$ is a general connection in $F Y \rightarrow M$. Let $\lambda^{1}$ be an $r$-th order linear connection in $V Y \rightarrow Y$. Using the construction $\Sigma(\Gamma, \Theta)$ from the previous section, we can construct a general connection $\mathcal{F}\left(\Xi, \lambda_{1}, \lambda\right)$ in $F Y \rightarrow Y$ as follows.

Let $Y^{2}=F Y \rightarrow Y^{1}=Y \rightarrow Y^{0}=M$ be the 2-fibred manifold. We have a general vertical connection $\Theta=\Theta\left(\lambda^{1}\right): Y^{2} \times_{Y^{1}} V^{10} Y^{1} \rightarrow V^{20} Y^{2}$ in $Y^{2} \rightarrow Y^{1} \rightarrow Y^{0}$ by

$$
\Theta\left(\lambda^{1}\right)\left(y^{2}, v^{1}\right):=\mathcal{F} X\left(y^{2}\right), j_{y^{1}}^{r}(X):=\lambda^{1}\left(v^{1}\right),
$$

$y^{2} \in Y_{y^{1}}^{2}, y^{1} \in Y^{1}, v^{1} \in V_{y^{1}}^{10} Y^{1}$, where $\mathcal{F} X$ is the flow lift of $X$ with respect to $F$. Denote $\Gamma=(\mathcal{F}(\Xi, \lambda), \Xi)$. Consequently, we have a general connection $\mathcal{F}\left(\Xi, \lambda, \lambda^{1}\right)$ in $F Y \rightarrow Y$ by

$$
\mathcal{F}\left(\Xi, \lambda, \lambda^{1}\right):=\Sigma\left(\Gamma, \Theta\left(\lambda^{1}\right)\right) .
$$

Let $\Xi$ and $\lambda$ be as above and $\Lambda$ be an $r$-th order linear connection on $Y$ (i.e. $r$-th order linear connection in $T Y \rightarrow Y$ ). Using the above construction $\mathcal{F}\left(\Xi, \lambda, \lambda^{1}\right)$, we can construct a general connection $\mathcal{F}(\Xi, \lambda, \Lambda)$ in $F Y \rightarrow Y$ as follows.

We have an $r$-th order linear connection $\lambda^{1}=\lambda^{1}(\Lambda, \Xi)$ in $V Y \rightarrow Y$ by

$$
\lambda^{1}(v)=j_{y}^{r}\left(p r^{\Xi} \circ X\right), j_{y}^{r} X:=\Lambda(v), v \in V_{y} Y, y \in Y,
$$


where $p r^{\Xi}: T Y \rightarrow V Y$ is the $\Xi$-projection. Then we have a general connection $\mathcal{F}(\Xi, \lambda, \Lambda)$ in $F Y \rightarrow Y$ by

$$
\mathcal{F}(\Xi, \lambda, \Lambda):=\mathcal{F}\left(\Xi, \lambda, \lambda^{1}(\Lambda, \Xi)\right)
$$

\section{REFERENCES}

[1] Kolářr, I., Michor, P. W., Slovák, J., Natural Operations in Differential Geometry, Springer-Verlag, Berlin, 1993.

Jan Kurek

Institute of Mathematics

Maria Curie-Skłodowska University

pl. M. Curie-Skłodowskiej 1

Lublin

Poland

e-mail: kurek@hektor.umcs.lublin.pl

Włodzimierz M. Mikulski

Institute of Mathematics

Jagiellonian University

ul. S. Łojasiewicza 6

Cracow

Poland

e-mail: Wlodzimierz.Mikulski@im.uj.edu.pl

Received November 9, 2015 